\title{
Autophagy induced by Helicobacter pylori infection is necessary for gastric cancer stem cell emergence
}

\author{
Sarah Courtois ${ }^{1} \cdot$ Maria Haykal $^{1} \cdot$ Clément Bodineau $^{3,4}$ • Elodie Sifré ${ }^{1} \cdot$ Lamia Azzi-Martin $^{1}$ • Armelle Ménard ${ }^{1}$. \\ Francis Mégraud $^{1,2} \cdot$ Philippe Lehours $^{1,2} \cdot$ Raúl V. Durán $^{3} \cdot$ Christine Varon $^{1} \cdot$ Emilie Bessède $^{1,2}$
}

Received: 20 January 2020 / Accepted: 28 July 2020 / Published online: 17 September 2020

(c) The International Gastric Cancer Association and The Japanese Gastric Cancer Association 2020

\begin{abstract}
Background The main cause of gastric cancer is the infection by the bacterium Helicobacter pylori which induces a chronic inflammation and an epithelial-to-mesenchymal transition (EMT) leading to the emergence of cells with cancer stem cell (CSC) properties. However, the underlying mechanisms have not been fully characterized. Moreover, H. pylori modulates the host cell autophagic process, but a few studies have investigated the role of this process in tumoral transformation. The aim of this study was to determine whether $\mathrm{H}$. pylori-induced autophagy has a role in CSC emergence.

Methods Autophagic flux in response to $H$. pylori infection was characterized in AGS cell line expressing the tandemtagged mCherry-GFP-LC3 protein and using a ratiometric flow cytometry analysis. Then, AGS and MKN45 cell lines were treated with bafilomycin or chloroquine, two pharmaceutical well-known inhibitors of autophagy, and different EMT and CSC characteristics were analyzed.

Results First, a co-expression of the gastric CSC marker CD44 and the autophagic marker LC3 in mice and human stomach tissues infected with $H$. pylori was observed. Then, we demonstrated in vitro that $H$. pylori was able to activate the autophagy process with a reduced autophagic flux. Finally, infected cells were treated with autophagy inhibitors, which reduced (i) appearance of mesenchymal phenotypes and migration ability related to EMT and (ii) CD44 expression as well as tumorsphere formation capacities reflecting CSC properties.

Conclusion In conclusion, all these data show that $H$. pylori-induced autophagy is implicated in gastric CSC emergence and could represent an interesting therapeutic target.
\end{abstract}

Keywords Autophagic flux $\cdot$ Epithelial-to-mesenchymal transition $\cdot$ CD44 $\cdot$ Tumorspheres $\cdot$ mTORC1

Electronic supplementary material The online version of this article (https://doi.org/10.1007/s10120-020-01118-9) contains supplementary material, which is available to authorized users.

Emilie Bessède

emilie.bessede@u-bordeaux.fr

1 Univ. Bordeaux, INSERM, BaRITOn, U1053, F-33000, Bordeaux, France

2 French National Reference Center for Campylobacters and Helicobacters (CNRCH), University Hospital of Bordeaux, Place Amelie Raba Leon, 33076 Bordeaux, France

3 Centro Andaluz de Biología Molecular Y Medicina Regenerativa-CABIMER, Consejo Superior de Investigaciones Científicas, Universidad de Sevilla, Universidad Pablo de Olavide, Américo Vespucio 24, 41092 Sevilla, Spain

4 Institut Européen de Chimie et Biologie, INSERM U1218, University of Bordeaux, Pessac, France

\section{Introduction}

Gastric cancer is the fifth most common cancer in the world and the third leading cause of cancer-related deaths worldwide [1]. Gastric adenocarcinoma (GC) has a poor prognosis with only one in five patients surviving longer than 5 years after diagnosis. The main known cause is the chronic infection with Helicobacter pylori (H. pylori). This bacterium has been classified as a Group I carcinogen by the World Health Organisation in 1994. This worldwide infection affects more than half of the world's population [2]: approximately $1 \%$ of infected people develop a gastric adenocarcinoma, reaching $10 \%$ in Japan. Elucidating the bacterial mechanisms implicated in gastric carcinogenesis will offer new potential therapeutics.

The previous studies have demonstrated that $H$. pylori induces an epithelial-to-mesenchymal transition (EMT) 
characterized by an elongated phenotype called "hummingbird", migratory and invasive properties, and the expression of specific EMT markers (Snail1, Twist, Zeb1, N-cadherin, Vimentin, etc.) [3]. This EMT induces emergence of cells with cancer stem cell (CSC) properties [4], characterized by self-renewal and asymmetrical division properties, resistance to conventional therapies (chemo- and radio-therapies), and thus responsible for tumor recurrence and metastasis [6, 7], highlighting the importance of targeting these cells. In GC, they express a high level of the cell-surface marker CD44 [5, $8,9]$, a major adhesion molecule for the extracellular matrix. Different variable isoforms exist and have been implicated as key players in malignant transformation. If the specific roles of each variant are not totally described, CD44v6 and CD44v9 were particularly studied and were respectively associated with advanced stages and described as a predictive marker for gastric cancer recurrence [10-12]. However underlying mechanisms leading to gastric CSC emergence in response to H. pylori infection are not fully defined.

Autophagy is a cellular physiological process used to degrade intracellular components, providing energy under starvation conditions or cellular stress [13]. In cancer, autophagy has a dual role: on one hand, it has a tumor-suppressive function by inducing autophagic death in tumoral cells [14], and on the other hand, it can promote tumoral transformation and chemoresistance [15, 16]. Indeed, autophagy was previously shown to contribute to stemness maintenance of CSC by promoting the expression of stem cell markers, providing energy and nutrients for CSCs.[17]. In GC, a link between autophagic-related protein expression (LC3, Beclin 1, and AMBRA (1) and prognosis factor has recently been demonstrated. Patients with immunopositive autophagic signature exhibited the worst prognosis [18]. Thus, targeting autophagy in GC could represent an interesting strategy, as different autophagy inhibition strategies improve anticancer therapies' efficiency [19-24]. However, other studies have also shown that autophagy inhibition can promote cancer in certain cases, reflecting the dual role of autophagy with both tumor promoter and tumor suppressor functions [25-27]. It is, therefore, important to characterize the role of autophagy in GC, especially since $H$. pylori infection can modulate host cell autophagy $[28,29]$. In this context, the aim of this study was to characterize the role of H. pylori-regulated autophagy in gastric tumoral transformation and particularly on CSC emergence.

\section{Materials and methods}

\section{Gastric cancer cell lines and treatments}

The gastric epithelial cell lines AGS, AGS-LC3, and MKN45 were cultured in DMEM/F12 media (AGS) or
RPMI-1640 media (MKN45) (Invitrogen, Cergy-Pontoise, France) supplemented with GlutaMAX, 10\% heatinactivated fetal bovine serum, and vancomycin $(50 \mu \mathrm{g} /$ ml) (Sigma, Saint-Quentin Fallavier, France), at $37{ }^{\circ} \mathrm{C}$ in a humidified $5 \% \mathrm{CO}_{2}$ atmosphere.

Cell lines were treated with $0.1 \mu \mathrm{M}$ bafilomycin or $100 \mu \mathrm{M}$ chloroquine (Sigma) for $3 \mathrm{~h}$ before the end of the experiment to study the autophagic flux or for $24 \mathrm{~h}$, concomitantly with infection, to inhibit the autophagy process.

\section{Lentiviral transduction}

Lentiviral vectors allowing the production of a tandem labeled mCherry-GFP-LC3 were kindly provided by Dr Mergny-Djavahery. Lentiviruses were used to infect the cells at a multiplicity of infection (MOI) of one in RPMI medium with $10 \mathrm{mM}$ HEPES. After $24 \mathrm{~h}, 2 \mu \mathrm{g} / \mathrm{mL}$ of puromycin (Sigma) were added to the medium to select transduced cells. Cells were amplified and mCherry-positive cells were selected by fluorescence-activated cell sorting using a BD FACSAria $^{\text {TM }}$ III sorter (Becton Dickinson, Franklin Lakes, New Jersey, USA) at the University of Bordeaux's cytometry platform. Purified AGS cells expressing mCherry-GFP-LC3 were named AGS-LC3 cell line.

\section{Bacterial culture}

H. pylori strains $(7.13, \mathrm{P} 12$, and SS1) were cultured at the National Reference Center for Campylobacters and Helicobacters of Bordeaux (CNRCH) as previously described [30]. Cells infections were performed at MOI 25.

\section{Transcriptomic analysis}

AGS and MKN45 cell lines were infected for $24 \mathrm{~h}$ with H. pylori 7.13. RNAs were extracted using RNeasy (Qiagen), the RNA Integrity Number (RINs) were determined on the TapeStation (Agilent, Les Ulis, France) and extracted RNAs were quantified by their absorbance at $260 \mathrm{~nm}$. The transcriptomic analysis was performed with the G3 HE $8 \times 0$ microarrays (Agilent), at the GeTrix Platform (Toulouse, France). Gene expression was analyzed using the KEGG pathway database.

\section{Immunoblots}

After the respective infections and/or treatments, cells were lysed with RIPA buffer composed with protease inhibitor (P8340) (Sigma), phosphatase inhibitors (P0044 and P5726) (Sigma), and PMSF (1 mM). After the electrophoresis process, proteins were transferred to a nitrocellulose membrane (Midi kit, Bio-Rad, Marnes-la-Coquette, France) using a 
Trans-Blot Turbo Transfer System (Bio-Rad). Membranes were imaged using a ChemiDoc MP imager (Bio-Rad).

Antibodies against S6 (\#2217, dilution 1:1,000), phospho-S6 (Ser235/236) (\#4858, dilution 1:1,000), S6K (\#2708, dilution 1:1,000), phospho-S6K(T389) (\#9205, dilution 1:1,000), and LC3A/B (\#12,741, dilution 1:1,000) were obtained from Cell Signaling Technology (Leiden, The Netherlands). Antibody against $\alpha$-tubulin (T9026, dilution 1:5,000) was obtained from Sigma. The secondary antibodies anti-mouse (\#7076, dilution 1:1,000) and anti-rabbit (\#7074, dilution 1:1,000) were obtained from Cell Signaling Technology.

\section{Ratiometric flow cytometry and flow cytometry analysis}

After infections and/or treatments, cells were resuspended in PBS with $0.5 \%$ bovine serum albumin and $2 \mathrm{mM}$ EDTA buffer. Autophagic flux was directly measured as previously described [31, 32]. The appropriate forward/side scatter profile was used to exclude non-viable cells. All mCherry-positive cells were included in the gate to measure the mCherry/ GFP ratio, with a scaling of $25 \%$. For CD44 quantification, cells were stained with anti-CD44-APC antibody (1:25 each, BD Pharmingen, San Jose, CA, USA). Flow cytometry experiments were performed using BD LRSFortessa and BD FACS CantoII instruments, and DIVA software (both from Becton Dickinson, Le Pont de Claix, France). Results were based on the analysis of 10,000 cells.

\section{Scratch wound healing assay}

Cells were seeded in 24-well plates. After $24 \mathrm{~h}$, scratches were performed and cells were infected and/or treated. Migration was evaluated by measuring the area without cells at 0 and $24 \mathrm{~h}$ after scratching using the MRI Wound Healing Tool (ImageJ) and the percentage of wound closure was calculated.

\section{Immunofluorescence technique}

Cells were cultured on glass coverslips and fixed with $4 \%$ paraformaldehyde solution for $10 \mathrm{~min}$ and processed for immunofluorescent staining using primary and secondary antibodies at the following concentrations: 1:100 for rabbit anti-Twist (Santa-Cruz Biotechnology), rabbit antiSnail1 (Santa- Cruz Biotechnology), rabbit anti-phosphoS6 (Ser235/236) (Cell Signaling), and 1:300 for anti-rabbit Alexa-488-labeled secondary antibodies. Alexa-546-labeled phalloidin (1:300) and DAPI (1:100) were used for F-actin and nuclear staining, respectively (all from Molecular Probes, Invitrogen, Cergy-Pontoise, France). Images were taken using an Eclipse 50i epi-fluorescence microscope
(Nikon Instruments, Champigny sur Marne, France) with Nis Element acquisition software.

\section{Tumorsphere formation}

Twenty-four hours after the treatments, cells were seeded in non-adherent plates (previously coated with a $10 \%$ poly-2-hydroxyethylmathacrylate (polyHEMA, Sigma), in a serum-free medium, and the number of tumorspheres formed was determined after 6 days.

\section{In vivo material}

Stomachs from mice were recovered from a previous study [33]. C57BL/6 mice of 6 weeks of age were infected with H. pylori SS1 or PBS by oral gavage for 3 days. Stomachs were collected after 12 months of infection. Mice experiments were performed in level 2 animal facilities in Bordeaux University with the approval of the local Ethical Committee, and in conformity with the French Ministry of Agriculture (approval number 4608).

\section{Histology and immunohistochemistry}

Immunohistochemistry (IHC) protocols were performed on serial tissue Sects. ( $1 \mu \mathrm{m}$ thick) from formalin-fixed paraffin-embedded stomachs, using rat anti-CD44 (1:200, IM7, E-Biosciences), rabbit anti-LC3 antibodies (1/500, D3U4C, Cell Signaling), and rabbit anti-Helicobacter pylori (1/300, B0471, Dako). A periodic acid Schiff staining was also performed on the serial tissue sections as usually described.

\section{Statistical analysis}

Data were expressed as the mean and the interval of values. Kruskal \& Wallis test or one-way analysis of variance (ANOVA) for multiple comparisons was performed. Differences were considered significant at $p<0.05$.

\section{Results}

\section{CD44 and LC3 expression are co-expressed in stomach tissues from mice infected with $H$. pylori}

Since CD44 is considered as a gastric CSC marker, and the microtubule-associated protein light chain (LC3) is used to monitor autophagic induction, both CD44 and LC3 stainings were performed on stomach tissues from H. pylori-infected or uninfected mice (Fig. 1). In uninfected mice, gastric epithelial cells did not express CD44 and a weak LC3 expression is observed, revealing the basal level of autophagy (Fig. 1a, b). In H. pylori-infected 
mice, metaplasia lesions detected by mucin-producing cells ( $\mathrm{f}-\mathrm{l}$ ) were observed, corresponding to clear cells, swollen glands with a high CD44 expression (Fig. 1d, g, j) and a higher LC3 expression (Fig. 1e, h, k). As both CD44 and LC3 expressions were simultaneous increased and co-expressed in infected gastric glands, a link between H. pylori infection, a regulation of autophagy pathway, and the emergence of gastric CSC can be evoked. This observation was also confirmed in 2 human cases where H. pylori infection was associated with an increase in the expression of CD44 and LC3 in the gastric epithelial cells (Supplementary Fig. 1).

\section{H. pylori infection induces autophagy in gastric cancer cells}

A transcriptomic analysis was performed on AGS and MKN45 cell lines infected or not with $H$. pylori. Except for $A T G 4 C$, all other significantly-regulated genes implicated in autophagy pathway were upregulated (Fig. 2a), suggesting an activation of the autophagy pathway in response to H. pylori infection. Among them, ULK1 (Unc51 Like Autophagy Activating Kinase 1, hATG1) codes for a Serine/Threonine kinase which induces autophagosome formation $[34,35]$ via the activation of the class III phosphatidylinositol 3-kinase (PIK3C3) complex, composed of the Activating Molecule in BECN1-Regulated Autophagy Protein 1 (AMBRA1) and the scaffold protein Beclin-1, also overexpressed in response to $H$. pylori infection (Fig. 2a).

ULK1 is a downstream effector of the mammalian target of rapamycin complex 1 (mTORC1), but also a negative regulator of this signaling pathway [36]. The transcriptomic analysis showed that the expression of downstream effectors positively regulated by mTORC1 (EIF4EBP2, EIF4E, and $R P S 6 K B 1$ ) was significantly decreased upon $H$. pylori infection (Supplementary Fig. 2a). Moreover, the phosphorylation of S6K and S6 (two well-known targets of mTORC1) was strongly decreased specifically in infected cells (Supplementary Fig. $2 b, c)$. All these results revealed that $H$. pylori infection inhibited the mTORC1 pathway in GC cell lines and could explain the increased expression of ULK1.

As ULK1 activation induces autophagosome formation, LC3 expression was studied considering that LC3-II is an indicator of the quantity of autophagosomes and used to monitor autophagic activity [37]. In response to $H$. pylori infection, the levels of LC3-II were strongly increased in both cell lines (Fig. 2b) and correlated with an increase in the autophagosome number in AGS-LC3 cells expressing the tandem-tagged mCherry-GFP-LC3 protein (Fig. 2c, d). All these results indicate that $H$. pylori infection induced autophagy in GC cell lines.

\section{The autophagic flux is reduced in response to $H$. pylori infection}

To characterize the autophagic flux induced in response to H. pylori infection, the mCherry/GFP ratio was measured using a ratiometric flow cytometry analysis in AGS-LC3 cells expressing the tandem-tagged mCherry-GFP-LC3 protein [31, 32]. As LC3-II is linked to mCherry and GFP, both fluorescences are detected in autophagosomes (Supplementary Fig. 1A). When autophagosomes merge with lysosomes to form autolysosomes, this compartment becomes acidic leading to the loss of the GFP fluorescence, which is sensitive to $\mathrm{pH}$ acidification. Therefore, only mCherry fluorescence is detected in autolysosomes (Supplementary Fig. 3A). The mCherry/GFP fluorescence ratio allowed us to distinguish a high from a low autophagic flux, and, therefore, a complete from an incomplete autophagy process (Supplementary Fig. 3A). When cells have a low autophagic flux, the mCherry/GFP ratio is low due to the presence of both fluorescence, whereas for cells with a high autophagic flux, the mCherry/GFP ratio is increased due to the loss of GFP fluorescence (Fig. 3a). To validate our model, AGSLC3 cells were cultured in Earle's Balanced Salt Solution (EBSS) medium. This nutrient restricted medium induces an increase of the autophagic flux in cells which will undergo a complete autophagy process to survive. Compared to the control condition (gray curve, Fig. 3a), in which cells were cultured in normal medium, the red curve representing cells cultured in EBSS shifted to the right and the mCherry/GFP ratio was increased from 125 to 150 (Fig. 3b), revealing an increase of the autophagic flux and validating the model.

In response to $24 \mathrm{~h}$ of infection with $H$. pylori 7.13 or P12, the mCherry/GFP ratio was significantly decreased by $\sim 20 \%$ and $\sim 40 \%$ respectively, compared to uninfected and untreated cells (Fig. 3b). This effect was visible by the shift to the left of the curve representing $H$. pylori-infected cells (yellow curve) in Fig. 3a, meaning that $H$. pylori infection reduced the autophagic flux.

To determine if the autophagic flux was totally blocked or just partially reduced in response to $24 \mathrm{~h}$ of infection, infected cells were treated with bafilomycin. This molecule blocks the fusion of autophagosomes with lysosomes leading to a total inhibition of the autophagic flux [38, 39]. When $\mathrm{H}$. pylori-infected cells were treated with bafilomycin, mCherry/GFP ratio was strongly decreased and reached 60, corresponding to a totally blocked and incomplete autophagy process. The bafilomycin-induced decrease in mCherry/GFP ratio in $H$. pylori-infected cells indicated that $H$. pylori infection only reduced the autophagic flux, which remains partially complete (hatched area, Fig. 3b). The same experiment was performed after a short time of infection $(4 \mathrm{~h})$ and the results were similar to those obtained after $24 \mathrm{~h}$ (Supplementary Fig. 3B). 

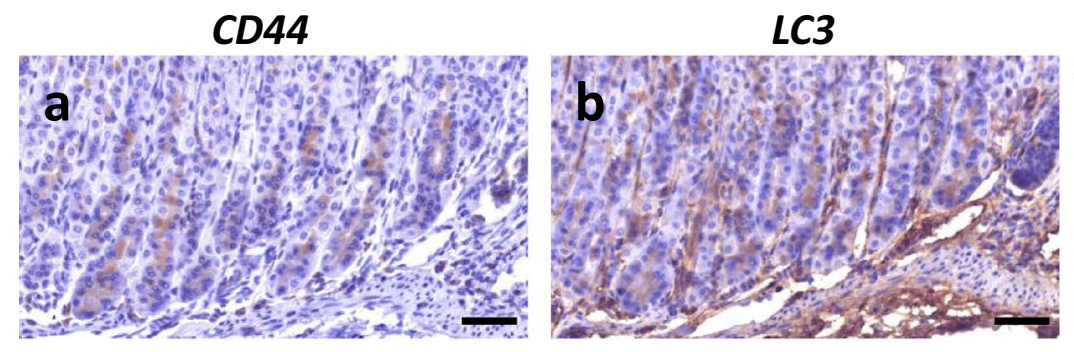

\section{LC3 \\ Scores}

PAS
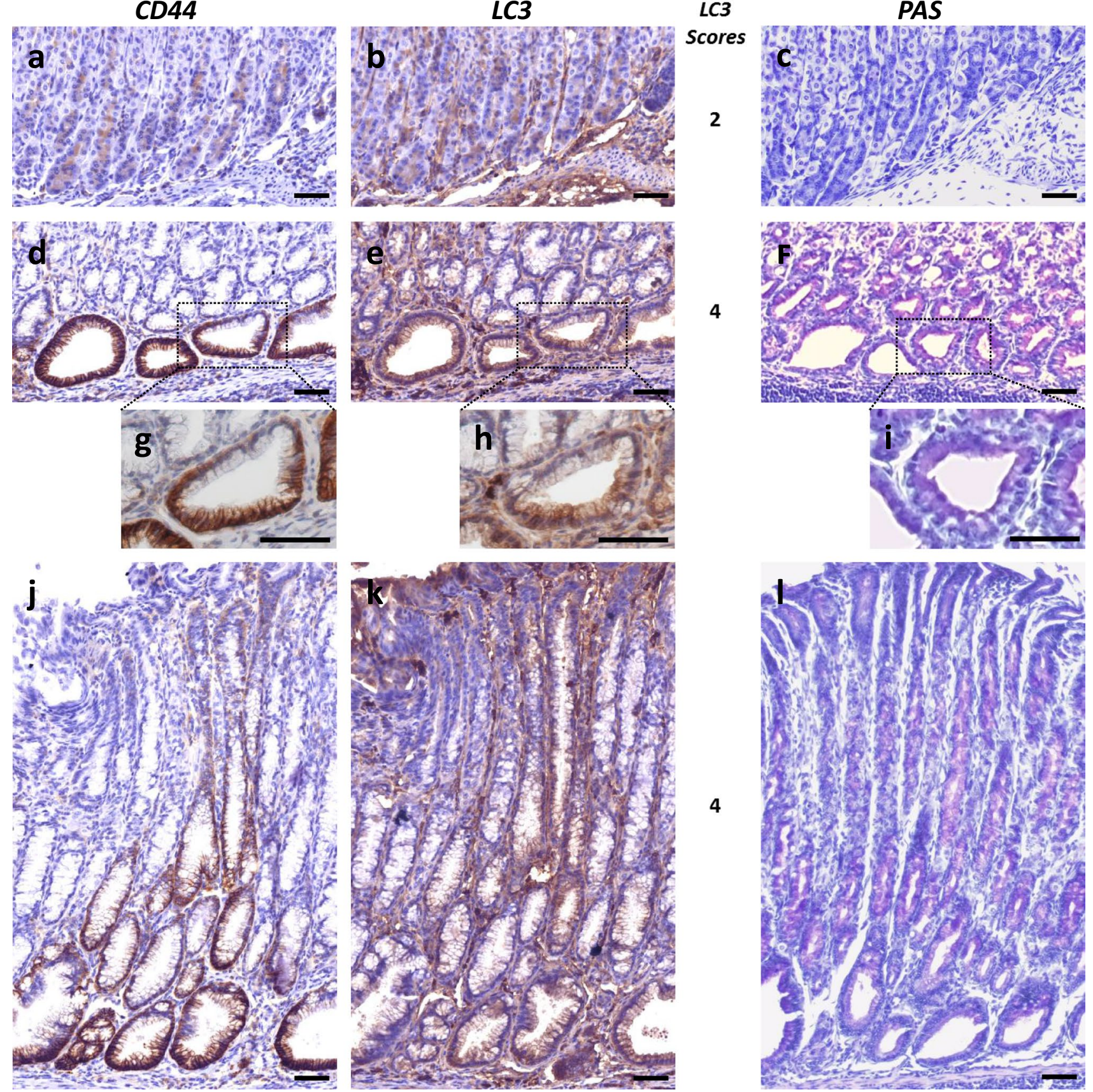

\section{4}
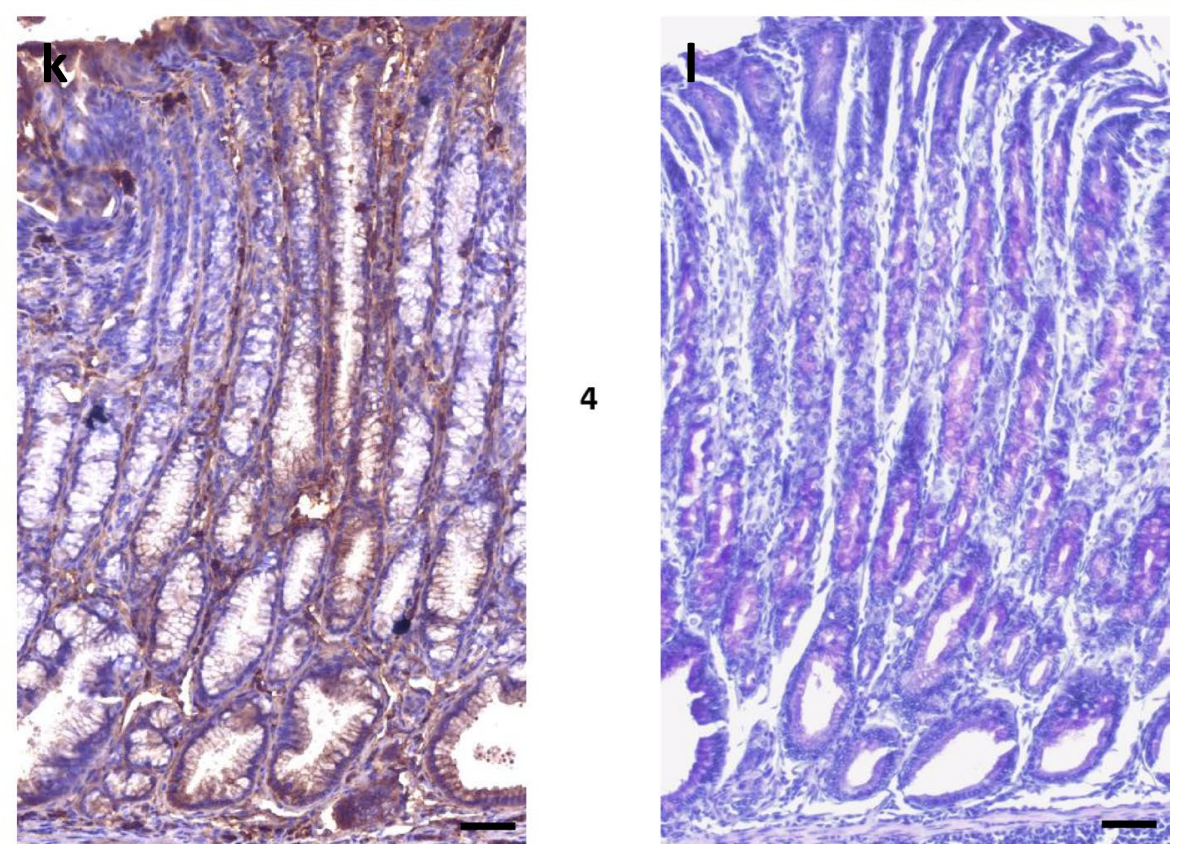

Fig. 1 CD44 and LC3 expressions are co-expressed in stomach tissue from mice infected with $H$. pylori. Representative images of histopathologic features of the gastric mucosa of uninfected $(\mathbf{a}, \mathbf{b}$, c) or H. pylori SS1-infected (d- 1 ) C57BL/6 mice after 12 months. a, b, c Normal gastric mucosa without CD44 expression (a), low LC3 expression (b), and no pink mucins staining (c). d-l Mucinous metaplasia lesions with swollen glands and clear cells, expressing CD44 $(\mathbf{d}, \mathbf{g}, \mathbf{j})$, LC3 (e, h, k), and mucins stained in pink by Periodic Acid

In summary, $H$. pylori infection increased the expression of autophagy-related genes, the amount of LC3-II protein, and the number of autophagosomes, revealing an induction of autophagy process with the infection. The
Schiff (PAS). g, h, i Higher magnification of a swollen gland with visible co-expressed of CD44 (g), LC3 (h) expressions, and mucins (i). Images are representative of the lesions obtained with $H$. pylori strain SS1 in C57BL/6 mice. LC3 scores were determined for each mouse (0: $0 \%$ of positive cells; $1:<10 \%$ positive cells; $2: 10-25 \%$ of positive cells; $3: 25-40 \%$ of positive cells; $4: 40-60 \%$ of positive cells; $5:>60 \%$ of positive cells). Scale bar $=100 \mu \mathrm{m}$

mCherry-GFP-LC3 model allowed us to demonstrate that $H$. pylori infection reduced the autophagic flux, meaning that a part of autophagy remains incomplete, without 
a

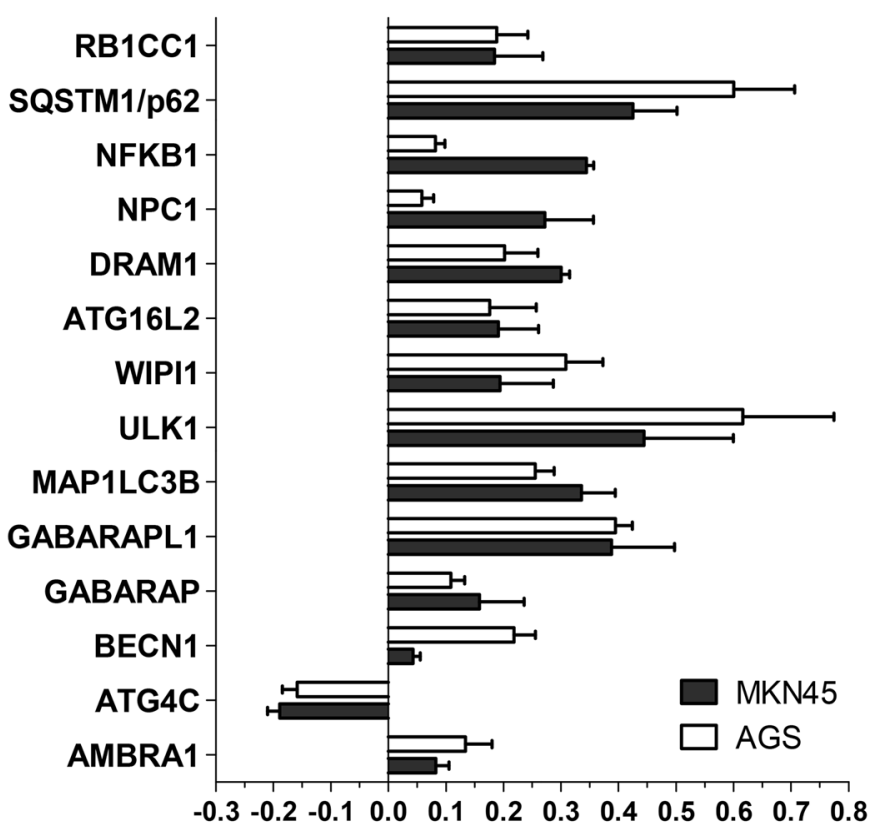

Log fold change relative to uninfected cells b

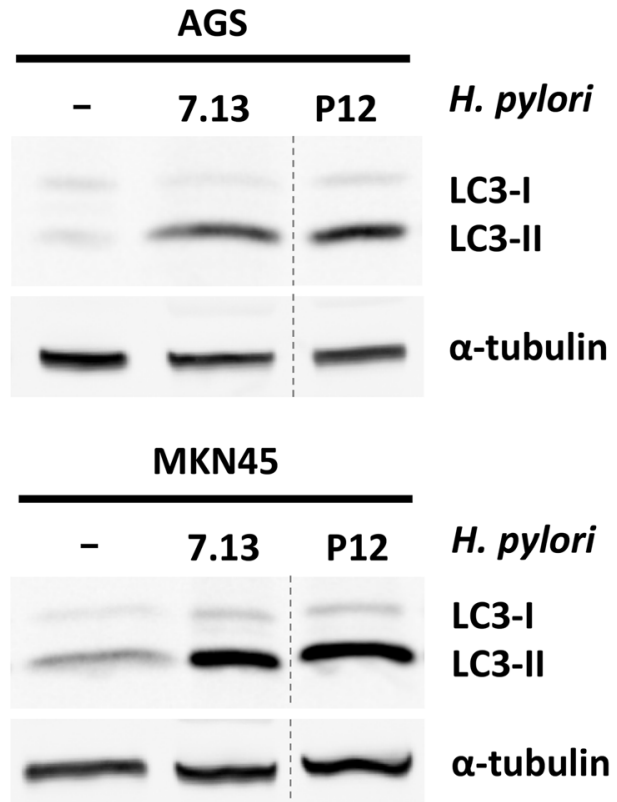

C

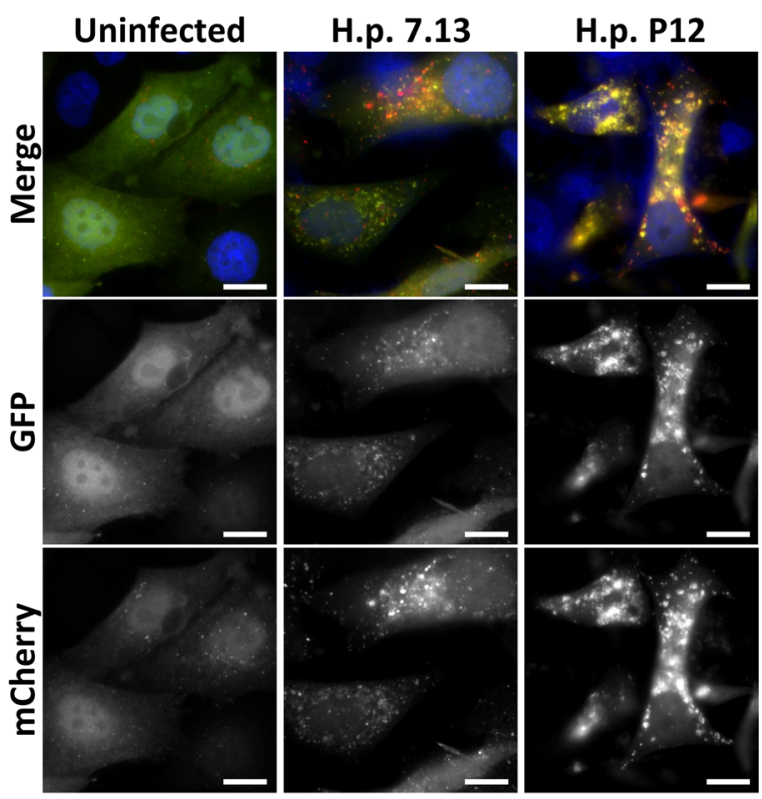

d

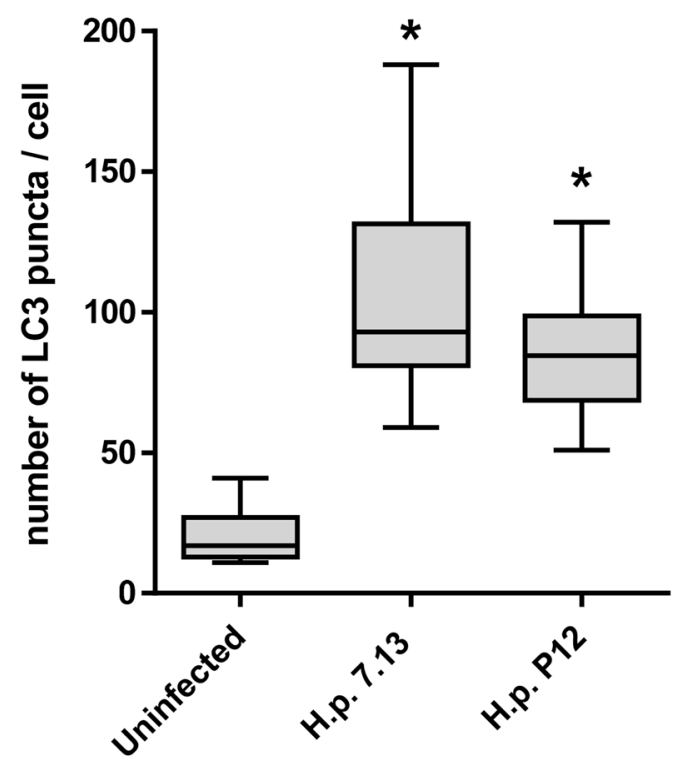

Fig. $2 \mathrm{H}$. pylori infection induces autophagy in gastric cancer cells. a, b AGS, MKN45, and AGS-LC3 cell lines were infected with $H$. pylori 7.13 or $\mathrm{P} 12$ for $24 \mathrm{~h}$. a mRNA relative expression of autophagy machinery components in both cell lines infected with H. pylori 7.13 compared to uninfected cells. Data represent signifi- cant variations observed in both cell lines $(n=4) . p<0.05$; Mann \& Whitney test. b Protein level of LC3 analyzed by western blot. c Images of representative AGS-LC3 cells. Bar scale: $10 \mu \mathrm{m}$. d LC3 puncta quantification on AGS-LC3 cells ( $n=2$ on 30 cells). $p<0.05$; Kruskal-Wallis with Dunn's post-test 


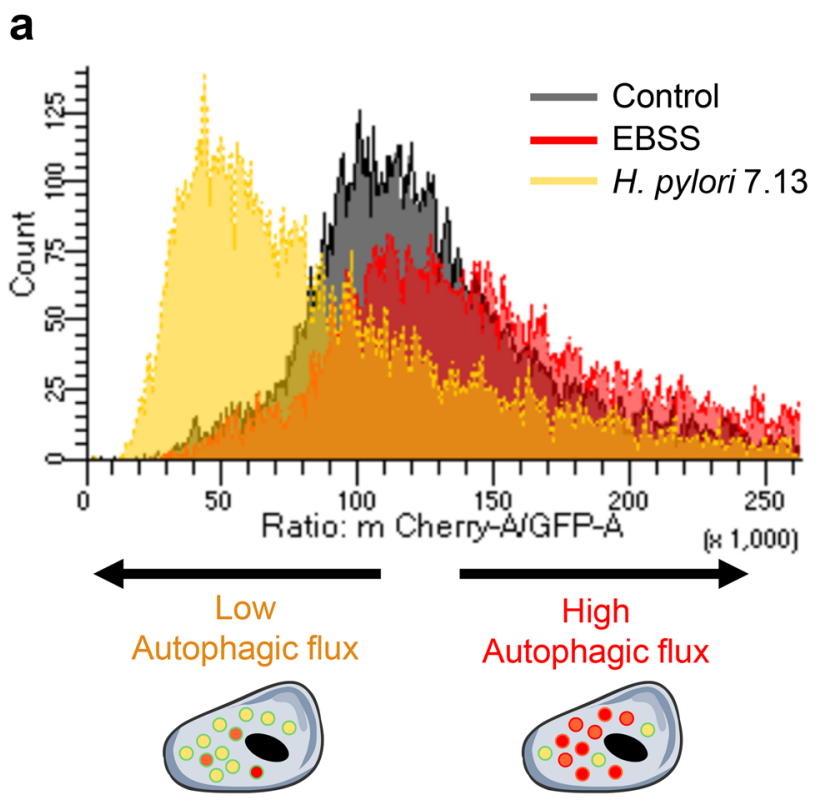

Fig. 3 The autophagic flux is reduced in response to H. pylori infection. AGS-LC3 cells, expressing mCherry-GFP-LC3, were infected or not with $H$. pylori 7.13 or P12 for $24 \mathrm{~h}$ and treated or not with bafilomycin or EBSS medium $3 \mathrm{~h}$ before the experiment ended. a Representative curves of mCherry/GFP ratios in three populations: control (uninfected and untreated) in gray, EBSS-treated in red, and

content degradation, while another part of autophagy remains complete with content degradation.

\section{Autophagy process sustains the EMT-like phenotype induced by $\mathrm{H}$. pylori}

To understand the role of autophagy in the emergence of CSC properties in response to $H$. pylori infection, GC cell lines were infected with $H$. pylori and subsequently treated with the autophagy inhibitors bafilomycin and chloroquine. Then, different EMT and CSC characteristics were studied.

H. pylori-infected cells acquired an EMT-like phenotype characterized by an elongated phenotype, called "hummingbird", and an enhanced migration ability. In infected cells, bafilomycin and chloroquine treatments strongly and significantly decreased the percentage of elongated cells induced by $H$. pylori, and almost restored the epithelial phenotype (Fig. 4a, b). This experiment also performed in an intestinal cell line deleted or not for atg 7 confirmed that inhibiting autophagy avoids "hummingbird" phenotype in response to H. pylori (Supplementary Fig. 4A). The cell migration ability was evaluated by performing scratch wound healing assays. In infected cells, the percentage of wound closure is higher than in uninfected cells (Fig. 4c, d) and this effect is independent of an increase of proliferation (Supplementary Fig. 4B). Autophagy inhibition significantly decreased the percentage of wound closure, compared to infected and

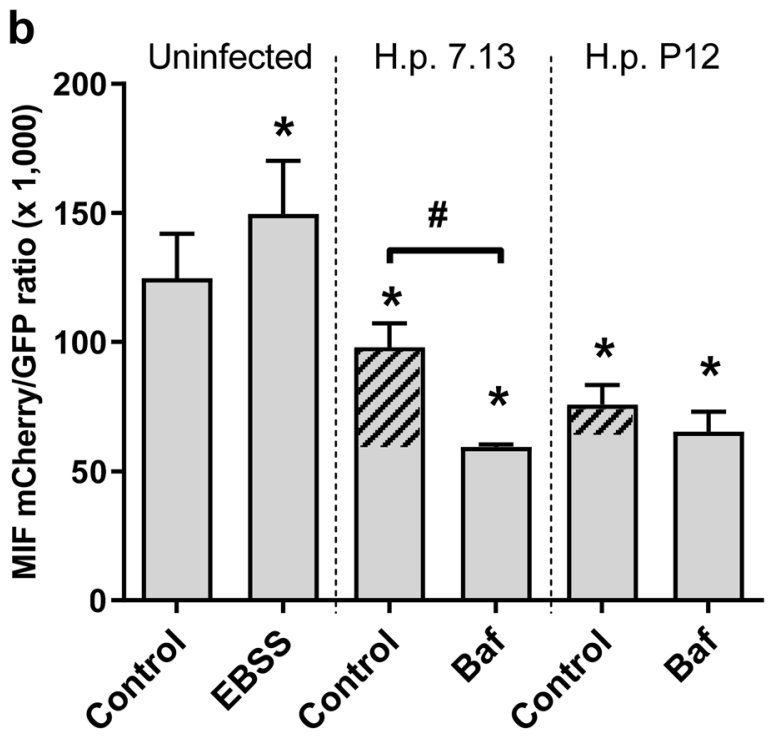

H. pylori 7.13 infected in yellow. b Mean of Fluorescence Intensity of mCherry/GFP ratios in AGS-LC3 cells $(n=6)$. Hatched area corresponds to the complete autophagy process in infected cells. ${ }^{*} p<0.05$ versus control; ${ }^{\#} p<0.05$ versus untreated condition; Oneway ANOVA with Bonferroni post-test. H.p. Helicobacter pylori, Baf. bafilomycin

untreated conditions (Fig. 4c, d). The EMT process induced by $H$. pylori is also characterized by an induction of the expression of EMT-related genes. Autophagy inhibition reduced the protein amount of Twist and Snail, without any gene expression variation (Supplementary Fig. 4C, D). In conclusion, these results indicated that $H$. pylori-induced autophagy was necessary for the EMT-like phenotype of the infected GC cells.

\section{Autophagy process is necessary for the emergence of cells with CSC properties in response to H. pylori infection}

Gastric CSCs are characterized by a high CD44 expression. In AGS-infected cells, a significant increase of the CD44 protein amount was observed in response to the infection. In MKN45 cells, the amount of CD44 was already very high and no significant increase was observed. In both cell lines, the inhibition of autophagy significantly decreased the amount of CD44 protein in infected cells (Fig. 5a).

A functional property of CSC is to survive and to form tumorspheres in non-adherent conditions, reflecting their ability to form tumors in vivo. This property is used to functionally quantify the CSC in vitro. To study the role of autophagy in the emergence of CSC-like cells, both cell lines were infected and/or treated with autophagy inhibitors under adherent culture conditions for $24 \mathrm{~h}$, prior to being seeded 
a

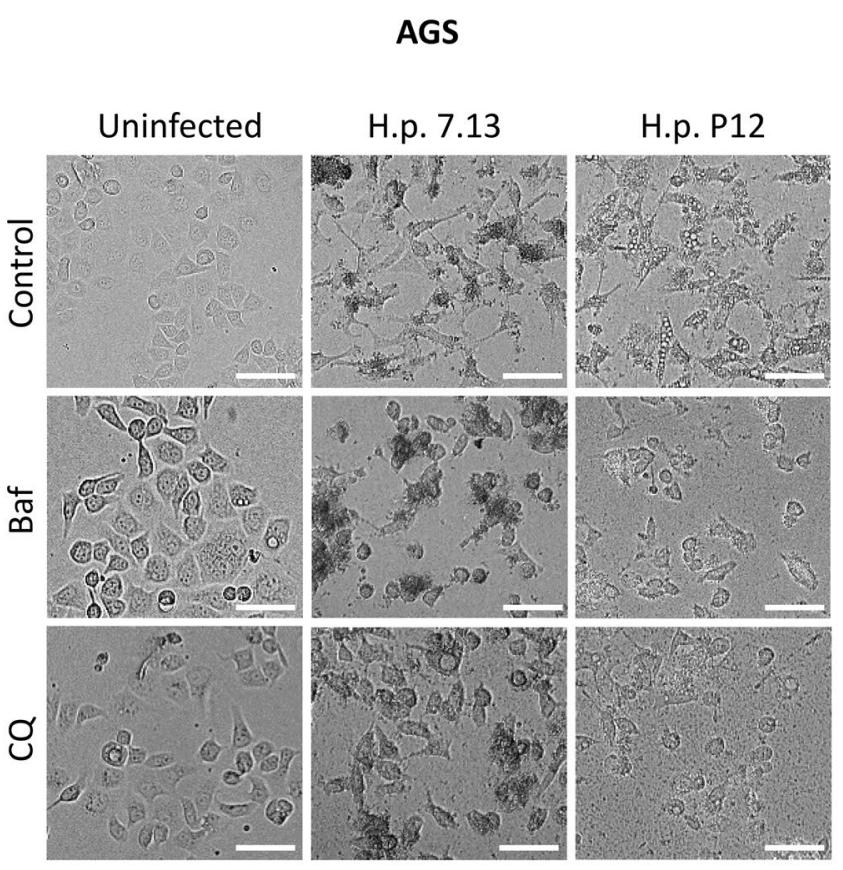

C

AGS

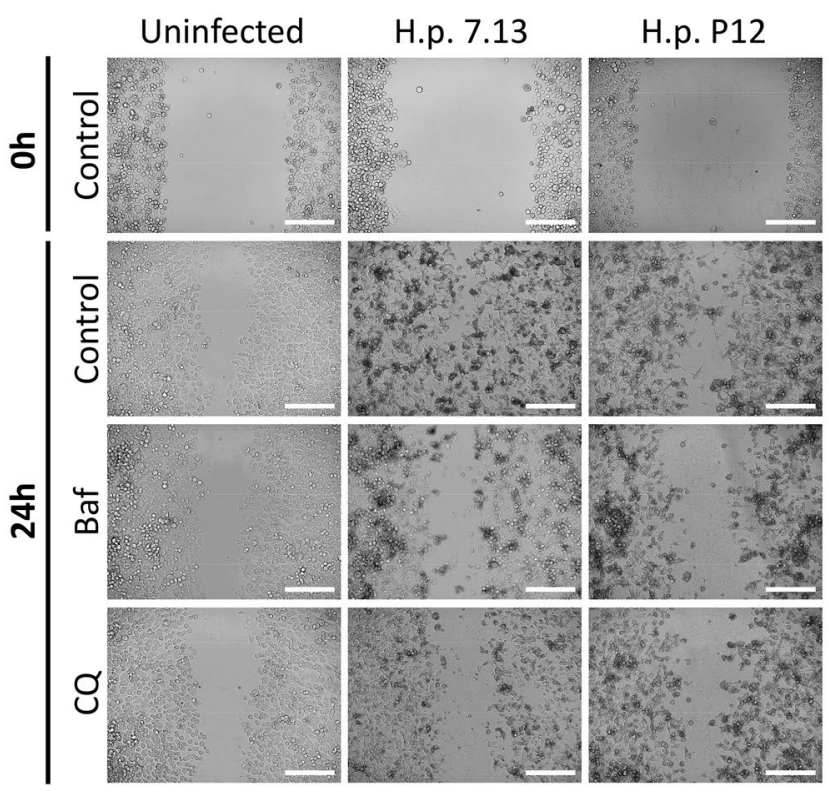

b

AGS

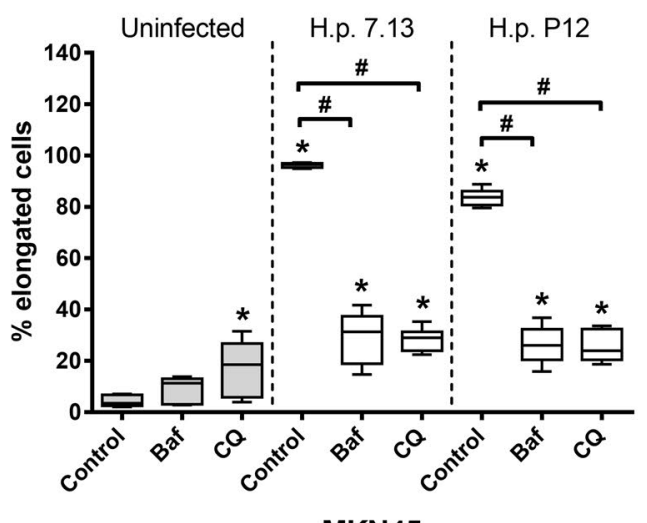

\section{MKN45}

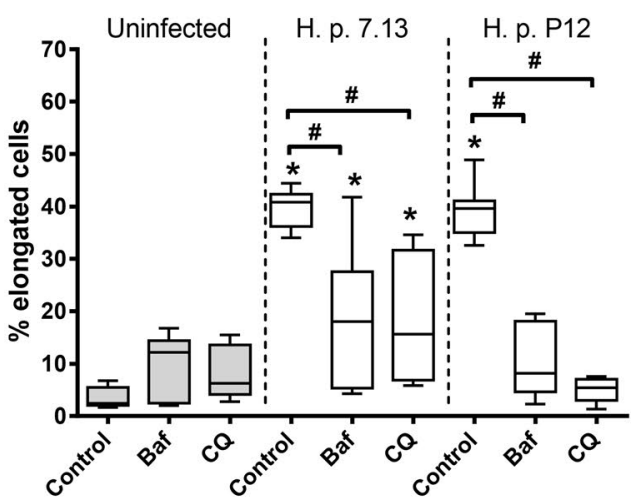

d

AGS

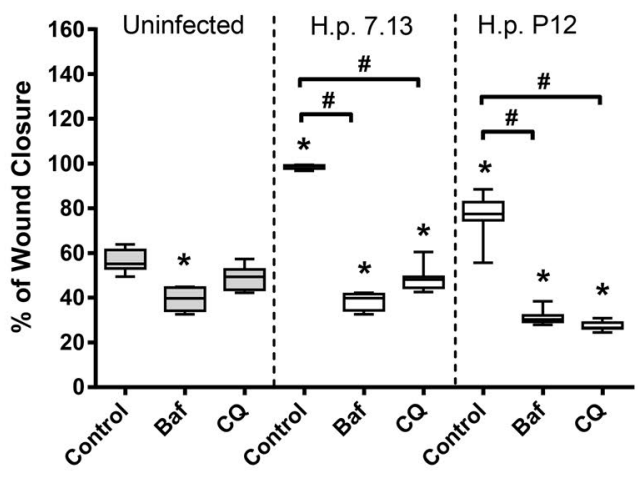

MKN45

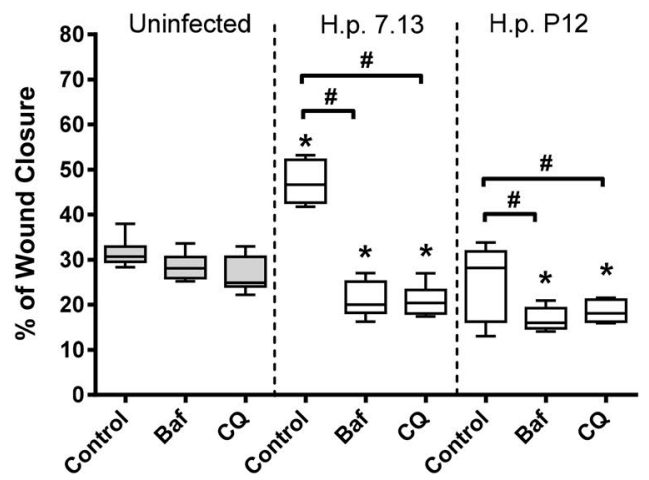


4Fig. $4 \mathrm{H}$. pylori-induced autophagy is necessary for hummingbird phenotype induction and migration ability. a, b AGS and MKN45 cells were infected or not with H. pylori 7.13 or P12 and treated or not with bafilomycin or chloroquine for $24 \mathrm{~h}$. a Representative images of AGS cells $24 \mathrm{~h}$ after infection and/or treatment. b Quantification of the hummingbird phenotype (percentage of elongated cells compared to normal cells). c, d Wound healing test performed on AGS and MKN45 cell lines. Cells were scratch-wounded, and then, cells were infected or not with $H$. pylori 7.13 or P12 and treated at the same time with or without bafilomycin (Baf, $0.1 \mu \mathrm{M})$ or chloroquine $(\mathrm{CQ}, 100 \mu \mathrm{M})$. $\mathbf{c}$ Representative images of AGS cells recorded immediately after the scratch and $24 \mathrm{~h}$ post-scratching. d Percentage of wound closure $24 \mathrm{~h}$ post-scratching. Data represent the mean and the interval between values $(n=3)$. ${ }^{*} p<0.05$ versus control; ${ }^{*} p<0.05$ versus untreated condition; One-way ANOVA with Bonferroni posttest. H.p. Helicobacter pylori, Baf. bafilomycin, $C Q$ Chloroquine

in non-adherent culture conditions without any additional treatment. Infection with $H$. pylori significantly increased the number of tumorspheres in both cell lines, compared to uninfected cells. Bafilomycin and chloroquine pretreatments significantly and strongly decreased the number of tumorspheres formed by infected cells (Fig. 5b, c), showing that $H$. pylori-induced autophagy is necessary for CSC emergence. Furthermore, tumorsphere size seemed to be reduced with autophagy inhibitor pretreatments (Fig. 5b), suggesting that $H$. pylori-induced autophagy is also implicated in CSC self-renewal capacities.

In summary, the inhibition of autophagy reduced the quantity of CD44 and the number of cells able to form tumorspheres, revealing that $H$. pylori-induced autophagy is a necessary process implicated in the emergence of cells with CSC properties in response to $H$. pylori infection.

\section{Discussion}

The aim of our study was to determine whether $H$. pyloriregulated autophagy is implicated in the $H$. pylori-dependent emergence of GC cells with CSC properties. First, CD44 and LC3 stainings on mice and human stomach tissues tended to be overexpressed and co-expressed in response to $H$. pylori infection. This first observation shows that a link between CSC emergence and autophagy could exist in the context of $H$. pylori infection. This idea was reinforced by different previous works which provided insights into the relationship between autophagy process and the emergence of CD44v9-positive cancer stem-like cells. Indeed, Tsugawa et al. recently reported that CagA accumulates in CD44v9positive gastric epithelial cells. These CD44v9-positive cells also overexpressed the Capping actin protein of muscle $Z$-line $\alpha$ subunit 1 (CAPZA1) which is able to indirectly inhibit autolysosome formation [40-42].

The study presented here was performed to investigate the functional link between $H$. pylori infection, autophagy induction, and gastric CSC emergence. Two gastric cancer cell lines, AGS and MKN45, were used in this work as they represent a good model to determine if the autophagy inhibition is implicated in the regulation of the EMT process in response to $H$. pylori, as these cells are predisposed to do EMT, due to their mutated E-cadherin status.

In response to $H$. pylori infection, expression of genes implicated in the autophagy process, LC3-II formation, and autophagosome number increased, showing that $H$. pylori infection induces autophagy as previously described [29]. Then, we demonstrated that $H$. pylori reduced the autophagic flux, leading to a partially incomplete autophagy without content degradation. The reason for this incomplete autophagy could be the accumulation of defective autophagosomes containing $H$. pylori, to escape exposure to antibiotics and allow its intracellular survival as previously proposed [43-45]. This effect could be dependent on VacA, as it was previously demonstrated that an exposure to VacA leads to an autophagosome maturation arrest [46].

With $H$. pylori infection, the autophagic flux was reduced but not totally blocked as a difference was observed in bafilomycin-treated cells compared to infected but untreated cells (Fig. 3 and Supplementary Fig. 3), meaning that a proportion of autophagy was, nevertheless, complete in response to the infection. This remaining complete autophagy could be implicated in the emergence of cells with CSC properties in response to the infection. To confirm this hypothesis, GC cell lines were infected and treated or not with bafilomycin or chloroquine, and EMT and CSC characteristics were studied.

Autophagy inhibition decreased the hummingbird phenotype, migration ability of infected cells, and decreased the amount of Twist and Snail mesenchymal markers. However, this effect seemed to be related to a post-transcriptional regulation as Twist and Snail gene expressions were not significantly reduced in response to autophagy inhibitor treatments. Moreover, blocking autophagy decreased the amount of CD44 protein and the tumorsphere number, related to the CSC number.

Thus, complete autophagy induced by $H$. pylori infection plays a pro-tumoral role and is necessary for EMT induction and emergence of cells with CSC properties. The relationship between $H$. pylori infection and autophagy induction on one hand [29], and between autophagy and CSC maintenance on the other hand [17], has already been established. However, this work is the first to establish a direct link between the $H$. pylori-regulated autophagy and the gastric CSC emergence, as the inhibition of autophagy in the context of the infection decreased the gastric CSC number. Furthermore, autophagy inhibitors showed no effect on uninfected cells, proving that it is the complete $H$. pylori-induced autophagy which is implicated in gastric CSC emergence. 
a

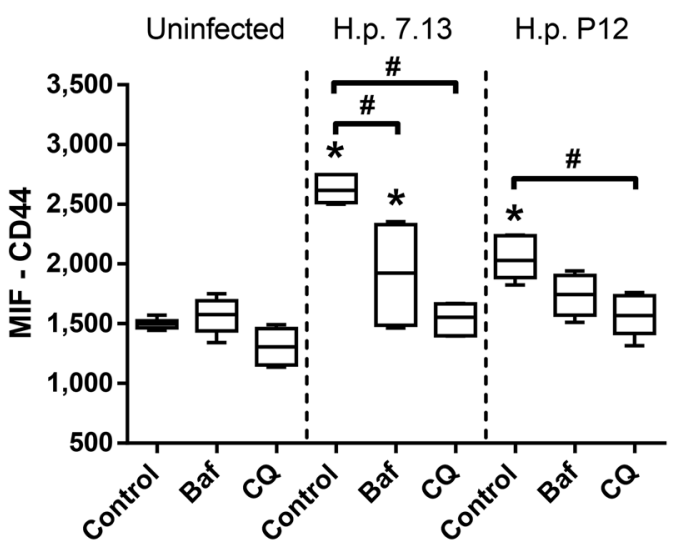

MKN45

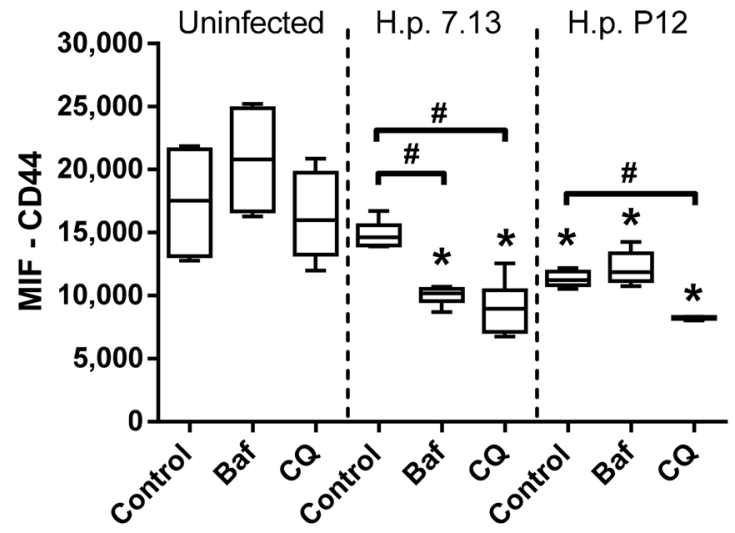

b

Uninfected

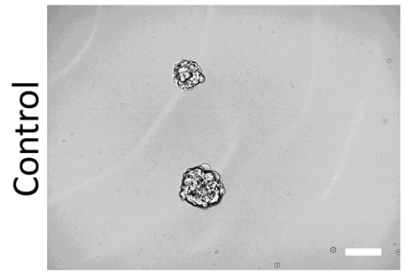

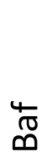
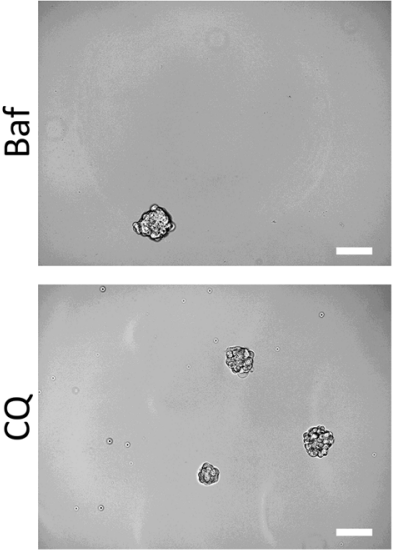

C

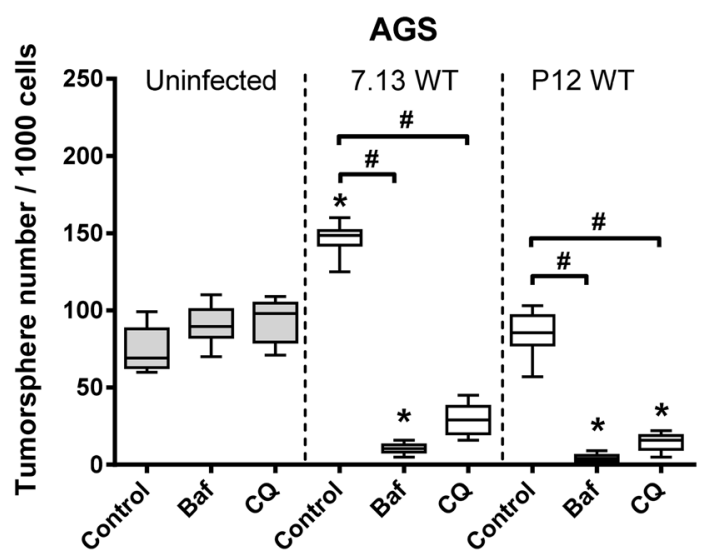

H.p. 7.13
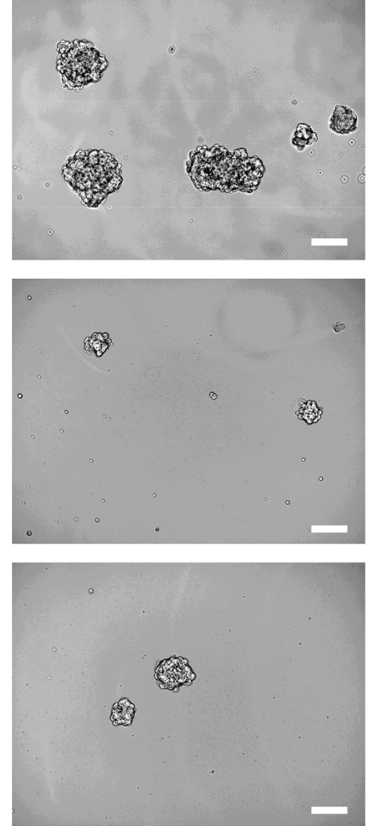

H.p. P12

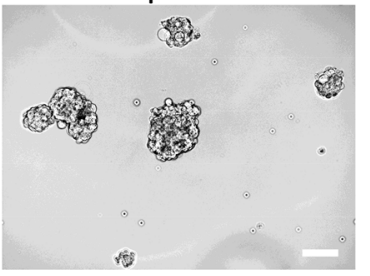

3
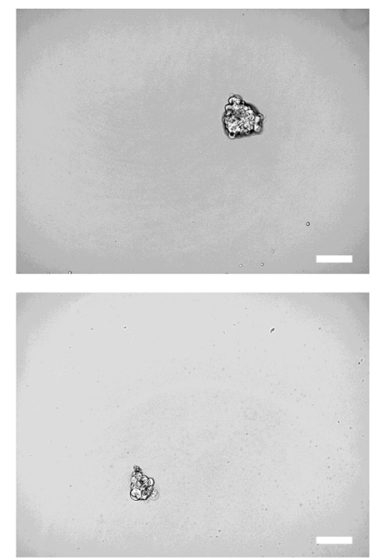

MKN45

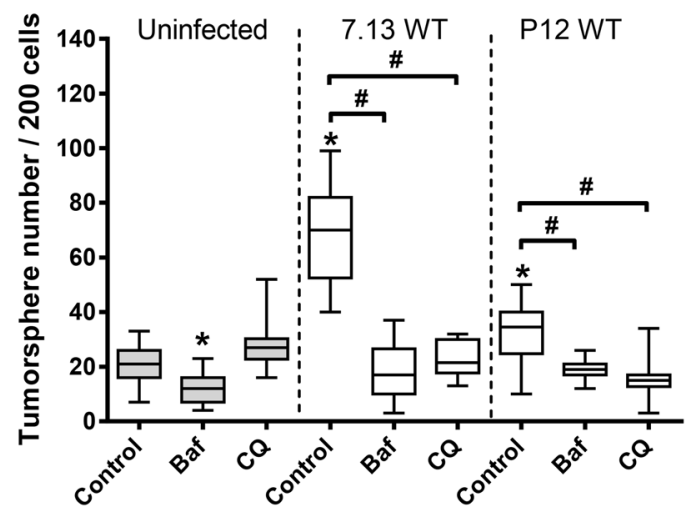


4 Fig. $5 \mathrm{H}$. pylori-induced autophagy is necessary for the emergence of CSC-like cells. AGS and MKN45 cells were infected or not with H. pylori 7.13 or P12 and treated or not with bafilomycin or chloroquine. a After $24 \mathrm{~h}$ of infection and/or treatment, cells were stained with anti-CD44-APC antibody and analyzed by flow cytometry. Data represent the mean of fluorescence intensity (MIF) and the interval of values $(n=3)$. ${ }^{*} p<0.05$ versus control; ${ }^{*} \mathrm{p}<0.05$ versus untreated condition; One-way ANOVA with Bonferroni post-test. b, c After $24 \mathrm{~h}$ cells from the different conditions were cultured under nonadherent conditions. b Representative images of MKN45 tumorspheres after 6 days. Bar scale: $100 \mu \mathrm{m}$. c Number of tumorspheres formed after 6 days. Data represent the mean and the interval of values $(n=3)$. ${ }^{*} p<0.05$ versus control; ${ }^{\#} p<0.05$ versus untreated condition; Kruskal \& Wallis with Dunn's post-test. H.p. Helicobacter pylori, Baf. bafilomycin, $C Q$ Chloroquine

Finally, virulence factors implicated in the autophagy regulation have not been clearly identified. The modulation of autophagy process in response to $H$. pylori infection is mostly attributed to the vacuolating toxin A (VacA) virulence factor [28, 29, 41, 42, 47]. However, the recent studies have shown that VacA is not the only factor responsible for autophagy modulation, as recent studies described that the $H$. pylori virulence factor gamma-glutamyltranspeptidase (HpGGT) impaired autophagic flux by disrupting lysosomal membrane integrity [48], and that CagA could also negatively regulate autophagy [49]. Further investigations are required to fully characterize the underlying mechanisms.

\section{Conclusions}

The present study was focused on the functional role of $H$. pylori-induced autophagy in gastric tumoral transformation and functionally demonstrated that this autophagy, induced by $H$. pylori, is necessary and sustains the EMTlike phenotype and the CSC features, placing autophagy as a potential mechanistic target to reduce $H$. pylori-induced tumorigenesis.

Acknowledgements We would like to thank Dr. Mojgan DjavaheriMergny and Dr. Jennifer Beauvarlet for kindly providing us the mCherry-GFP-LC3 lentivirus particles, and Wencan He for technical assistance. We would also like to thank Atika Zouine and Vincent Pitard for technical assistance at the Flow cytometry facility in Bordeaux (CNRS UMS 3427, INSERM US 005, University of Bordeaux, F-33000 Bordeaux, France).

Funding This work was supported by the French foundation Ligue contre le Cancer (Pyrénées Atlantiques).

\section{Compliance with ethical standards}

Conflicts of interest The authors declare no conflicts of interest.

\section{References}

1. Global Cancer Observatory [Internet]. [cited 2019 Jul 20]. Available from: https://gco.iarc.fr/

2. Hooi JKY, Lai WY, Ng WK, Suen MMY, Underwood FE, Tanyingoh D, et al. Global prevalence of Helicobacter pylori infection: systematic review and meta-analysis. Gastroenterology. 2017;153:420-9.

3. Kalluri R, Weinberg RA. The basics of epithelial-mesenchymal transition. J Clin Invest. 2009;119:1420-8.

4. Bessède E, Staedel C, Acuña Amador LA, Nguyen PH, Chambonnier L, Hatakeyama M, et al. Helicobacter pylori generates cells with cancer stem cell properties via epithelial-mesenchymal transition-like changes. Oncogene. 2014;33:4123-31.

5. Giraud J, Bessède E, Mégraud F, Varon C. Gastric cancer: a stem cell disease? 2017 [cited 2017 Sep 20]; Available from: https://www.intechopen.com/books/gastric-cancer/gastric-cance r-a-stem-cell-disease-

6. Reya T, Morrison SJ, Clarke MF, Weissman IL. Stem cells, cancer, and cancer stem cells. Nature. 2001;414:105-11.

7. Adams JM, Strasser A. Is tumor growth sustained by rare cancer stem cells or dominant clones? Cancer Res. 2008;68:4018-21.

8. Takaishi S, Okumura T, Tu S, Wang SSW, Shibata W, Vigneshwaran $\mathrm{R}$, et al. Identification of gastric cancer stem cells using the cell surface marker CD44. Stem Cells Dayt Ohio. 2009;27:1006-200.

9. Nguyen PH, Giraud J, Staedel C, Chambonnier L, Dubus P, Chevret E, et al. All-trans retinoic acid targets gastric cancer stem cells and inhibits patient-derived gastric carcinoma tumor growth. Oncogene. 2016;35:5619-28.

10. Hirata K, Suzuki H, Imaeda H, Matsuzaki J, Tsugawa H, Nagano $\mathrm{O}$, et al. CD44 variant 9 expression in primary early gastric cancer as a predictive marker for recurrence. Br J Cancer. 2013;109:379-86.

11. Xin Y, Grace A, Gallagher MM, Curran BT, Leader MB, Kay EW. CD44V6 in gastric carcinoma: a marker of tumor progression. Appl Immunohistochem Mol Morphol AIMM. 2001;9:138-42.

12. Zavros Y. Initiation and maintenance of gastric cancer: a focus on CD44 variant isoforms and cancer stem cells. Cell Mol Gastroenterol Hepatol. 2017;4:55-63.

13. Parzych KR, Klionsky DJ. An overview of autophagy: morphology, mechanism, and regulation. Antioxid Redox Signal. 2014;20:460-73.

14. Kroemer G, Levine B. Autophagic cell death: the story of a misnomer. Nat Rev Mol Cell Biol. 2008;9:1004-100.

15. Galluzzi L, Pietrocola F, Bravo-San Pedro JM, Amaravadi RK, Baehrecke EH, Cecconi F, et al. Autophagy in malignant transformation and cancer progression. EMBO J. 2015;34:856-80.

16. Liu J, Debnath J. The evolving, multifaceted roles of autophagy in cancer. Adv Cancer Res. 2016;130:1-53.

17. Lei Y, Zhang D, Yu J, Dong H, Zhang J, Yang S. Targeting autophagy in cancer stem cells as an anticancer therapy. Cancer Lett. 2017;393:33-9.

18. Ieni A, Cardia R, Giuffrè G, Rigoli L, Caruso RA, Tuccari G. Immunohistochemical expression of autophagy-related proteins in advanced tubular gastric adenocarcinomas and its implications. Cancers. 2019;11(3):389.

19. Xiong X, Lu B, Tian Q, Zhang H, Wu M, Guo H, et al. Inhibition of autophagy enhances cinobufagin-induced apoptosis in gastric cancer. Oncol Rep. 2019;41:492-500.

20. Zhao J, Nie Y, Wang H, Lin Y. MiR-181a suppresses autophagy and sensitizes gastric cancer cells to cisplatin. Gene. 2016;576:828-33. 
21. Zhang Q, Wu S, Zhu J, Chai D, Gan H. Down-regulation of ASIC1 suppressed gastric cancer via inhibiting autophagy. Gene. 2017;608:79-85.

22. Wang W, Liu L, Zhou Y, Ye Q, Yang X, Jiang J, et al. Hydroxychloroquine enhances the antitumor effects of $\mathrm{BC} 001$ in gastric cancer. Int J Oncol. 2019;55(2):405-14. https://doi.org/10.3892/ ijo.2019.4824.

23. Lin X, Peng Z, Wang X, Zou J, Chen D, Chen Z, et al. Targeting autophagy potentiates antitumor activity of Met-TKIs against Metamplified gastric cancer. Cell Death Dis. 2019;10:139.

24. Li W, Zhou Y, Yang J, Li H, Zhang H, Zheng P. Curcumin induces apoptotic cell death and protective autophagy in human gastric cancer cells. Oncol Rep. 2017;37:3459-66.

25. Chen P-P, Ma X-Y, Lin Q, Xu H-L, Shi H-X, Zhang H-Y, et al. Kangfuxin promotes apoptosis of gastric cancer cells through activating ER-stress and autophagy. Mol Med Rep. 2017;16:9043-50.

26. Yu Y-F, Hu P-C, Wang Y, Xu X-L, Rushworth GM, Zhang Z, et al. Paclitaxel induces autophagy in gastric cancer BGC823 cells. Ultrastruct Pathol. 2017;41:284-90.

27. Qin W, Li C, Zheng W, Guo Q, Zhang Y, Kang M, et al. Inhibition of autophagy promotes metastasis and glycolysis by inducing ROS in gastric cancer cells. Oncotarget. 2015;6:39839-54.

28. Terebiznik MR, Raju D, Vázquez CL, Torbricki K, Kulkarni R, Blanke SR, et al. Effect of Helicobacter pylori's vacuolating cytotoxin on the autophagy pathway in gastric epithelial cells. Autophagy. 2009;5:370-9.

29. Greenfield LK, Jones NL. Modulation of autophagy by Helicobacter pylori and its role in gastric carcinogenesis. Trends Microbiol. 2013;21:602-12.

30. Courtois S, Bénéjat L, Izotte J, Mégraud F, Varon C, Lehours $\mathrm{P}$, et al. Metformin can inhibit Helicobacter pylori growth. Future Microbiol. 2018;13:1575-83. https://doi.org/10.2217/ fmb-2018-0184.

31. Kimura S, Noda T, Yoshimori T. Dissection of the autophagosome maturation process by a novel reporter protein, tandem fluorescent-tagged LC3. Autophagy. 2007;3:452-60.

32. Gump JM, Thorburn A. Sorting cells for basal and induced autophagic flux by quantitative ratiometric flow cytometry. Autophagy. 2014;10:1327-34.

33. Bessède E, Molina S, Acuña-Amador L, Dubus P, Staedel C, Chambonnier L, et al. Deletion of IQGAP1 promotes Helicobacter pylori-induced gastric dysplasia in mice and acquisition of cancer stem cell properties in vitro. Oncotarget. 2016;7:80688-99.

34. Cao Y, Luo Y, Zou J, Ouyang J, Cai Z, Zeng X, et al. Autophagy and its role in gastric cancer. Clin Chim Acta Int J Clin Chem. 2018;489:10-20.

35. Russell RC, Tian Y, Yuan H, Park HW, Chang Y-Y, Kim J, et al. ULK1 induces autophagy by phosphorylating Beclin-1 and activating Vps34 lipid kinase. Nat Cell Biol. 2013;15:741-50.

36. Jung $\mathrm{CH}$, Seo M, Otto NM, Kim D-H. ULK1 inhibits the kinase activity of mTORC1 and cell proliferation. Autophagy. 2011;7:1212-21

37. Yoshii SR, Mizushima N. Monitoring and Measuring Autophagy. Int J Mol Sci. 2017;18:1865.
38. Yamamoto A, Tagawa Y, Yoshimori T, Moriyama Y, Masaki R, Tashiro Y. Bafilomycin A1 prevents maturation of autophagic vacuoles by inhibiting fusion between autophagosomes and lysosomes in rat hepatoma cell line, H-4-II-E cells. Cell Struct Funct. 1998;23:33-42.

39. Klionsky DJ, Elazar Z, Seglen PO, Rubinsztein DC. Does bafilomycin A1 block the fusion of autophagosomes with lysosomes? Autophagy. 2008;4:849-50.

40. Tsugawa H, Kato C, Mori H, Matsuzaki J, Kameyama K, Saya H, et al. Cancer stem-cell marker CD44v9-Positive cells arise from Helicobacter pylori-Infected CAPZA1-overexpressing cells. Cell Mol Gastroenterol Hepatol. 2019;8:319-34.

41. Tsugawa H, Suzuki H, Saya H, Hatakeyama M, Hirayama T, Hirata K, et al. Reactive oxygen species-induced autophagic degradation of Helicobacter pylori CagA is specifically suppressed in cancer stem-like cells. Cell Host Microbe. 2012;12:764-77.

42. Tsugawa H, Mori H, Matsuzaki J, Sato A, Saito Y, Imoto M, et al. CAPZA1 determines the risk of gastric carcinogenesis by inhibiting Helicobacter pylori CagA-degraded autophagy. Autophagy. 2019:15:242-58.

43. Capurro MI, Greenfield LK, Prashar A, Xia S, Abdullah M, Wong $\mathrm{H}$, et al. VacA generates a protective intracellular reservoir for Helicobacter pylori that is eliminated by activation of the lysosomal calcium channel TRPML1. Nat Microbiol. 2019;4(8):141123. https://doi.org/10.1038/s41564-019-0441-6.

44. Wang Y-H, Lv Z-F, Zhong Y, Liu D-S, Chen S-P, Xie Y. The internalization of Helicobacter pylori plays a role in the failure of $H$. pylori eradication. Helicobacter. 2017;22(1):e12324. https ://doi.org/10.1111/hel.12324.

45. Tang B, Li N, Gu J, Zhuang Y, Li Q, Wang H-G, et al. Compromised autophagy by MIR30B benefits the intracellular survival of Helicobacter pylori. Autophagy. 2012;8:1045-57.

46. Raju D, Hussey S, Ang M, Terebiznik MR, Sibony M, GalindoMata E, et al. Vacuolating cytotoxin and variants in Atg16L1 that disrupt autophagy promote Helicobacter pylori infection in humans. Gastroenterology. 2012;142:1160-71.

47. Yahiro K, Satoh M, Nakano M, Hisatsune J, Isomoto H, Sap J, et al. Low-density lipoprotein receptor-related protein-1 (LRP1) mediates autophagy and apoptosis caused by Helicobacter pylori VacA. J Biol Chem. 2012;287:31104-15.

48. Bravo J, Díaz P, Corvalán AH, Quest AFG. A novel role for Helicobacter pylori gamma-glutamyltranspeptidase in regulating autophagy and bacterial internalization in human gastric cells. Cancers. 2019;11(6):801.

49. Li N, Tang B, Jia Y-P, Zhu P, Zhuang Y, Fang Y, et al. Helicobacter pylori CagA protein negatively regulates autophagy and promotes inflammatory response via c-Met-PI3K/Akt-mTOR signaling pathway. Front Cell Infect Microbiol. 2017;7:417.

Publisher's Note Springer Nature remains neutral with regard to jurisdictional claims in published maps and institutional affiliations. 